\title{
The investigation of Poly(ADP-ribose)polymerase activity in the context of nucleosome
}

\author{
Kurgina T. ${ }^{1,2 *}$, Kutusov M. ${ }^{1,2}$, Belousova E. ${ }^{2}$, Naumenko K. ${ }^{2}$, Lavrik O. ${ }^{1,2}$ \\ ${ }^{1}$ Novosibirsk State University, Novosibirsk, Russia \\ ${ }^{2}$ Institute of Chemical Biology and Fundamental Medicine, SB RAS, Yekaterinburg, Russia \\ *e-mail: t.a.kurgina@gmail.com
}

Key words: PARP1, DNA repair, nucleosome

Poly(ADP-ribose)polymerase 1 (PARP1) - is a key enzyme that regulates the activity of DNA-repair machine. It makes PARP1 inhibitors promising anticancer drugs [1]. But some problems occur during therapy by these agents, for example cancer resistance or recurrence of disease. These problems require not only novel inhibitor developing, but also fundamental studies of PARP1 and its interaction with other proteins. To address this need we have developed a test-system, which allows analyzing PARP 1 activity in real time [2]. In this work we modified this test-system to observe PARP 1 activity on nucleosomes in vitro. Nucleosome is a DNA-protein complex that consist 147 bp DNA is wrapped around histone octamer. This model close to in vivo conditions more than the short model DNA, used previously. We showed the ability of our method shed light on PARP1 interaction with nucleosome, nucleosome with DNA-lesion and different proteins on nucleosome. PARP1 DNA-binding capacity after poly(ADP-ribose)lation and degradation of PAR by several enzymes (for example PARG) was studied. It is shown that automodified PARP1 can effectively bind DNA and DNA in nucleosome context after degradation of PAR. However, PARylation activity of this enzyme is dramatically decreased after modification and modification removing. In addition, an interaction of PARP1 with several nuclei proteins on nucleosome was studied. Some of these proteins can modulate PARP1 activity and cause decreasing of PARP1 inhibitors efficacy. Results of these studies show that the expression level of these proteins in patients can influence the choice of anticancer therapy strategy.

Acknowledgements: The work was supported by the grant from RSF 17-74-20075.

\section{References}

1. Jain P.G., Patel B.D. Medicinal chemistry approaches of poly ADP-Ribose polymerase 1 (PARP1) inhibitors as anticancer agents. European Journal Med. Chemistry. 2019;1(165):198-215.

2. Kurgina T.A., Anarbaev R.O., Sukhanova M.V., Lavrik O.I. A rapid fluorescent method for the real-time measurement of poly(ADP-ribose) polymerase 1 activity. Analytical Biochemistry. 2018;15(545):91-97. 